\section{FORMATION OF PLANETARY SYSTEMS}

A CONSIDERABLE amount of interest has been shown in recent times on the subject of the origin of planets and satellites, and various theories have been developed with the primary object of circumventing the angular momentum difficulty. A recent paper by B. M. Peek, entitled "The Formation of Planetary Systems" (J. Brit. Astro. Assoc., 53, 1 ; December, 1942), puts forward a theory which possesses at least the merits of simplicity. He starts off with a quotation from Sir James Jeans's "Astronomy and Cosmogony" (1928), which attempts to explain the angular momentum of the nebulæ: "Any currents or motion in the original medium would contribute angular momentum to the nascent nebulæ, and as these shrank to nebular dimensions, the constancy of angular momentum would result in fairly rapid rotations of the shrunken masses". Peek extends this view to the next order of condensation-the starsand is thus able to explain how the necessary angular momentum of the solar system, and other possible planetary systems toó, can be accounted for.

Starting with a portion of the galaxy having a nearly uniform distribution of matter, Peek postulates the beginning of the gravitational instability which produces stars, and further postulates that the random currents in the medium contribute angular momentum to these nascent stars. After a time any diffuse star rotating about an axis which is perpendicular to its equatorial or invariable plane will have most of its angular momentum concentrated close to the rim of this plane. The system has no resemblance to Laplace's nebula, which was supposed to rotate almost like a rigid body. For this reason the main bulk of the star will be almost spherical, not lenticular, as contraction proceeds. In time, however, the small portion of matter at the rim, which received its momentum from the original current, will have communicated some of its motion to other portions in the immediate vicinity, owing to viscosity. The shrinking of the star is necessarily accompanied by an increased angular velocity, in order that the angular momentum may be preserved, and finally portions of matter will be expelled from the periphery and will describe orbits about the still shrinking star. Some quantitative results are given, among which reference may be made to one in particular. Peek has calculated that if the original current had the same order of mass as all the planets combined and moved with a velocity of two metres per second, this current would have been able to endow the whole of the solar system with its observed angular momentum.

By the time all the matter had been shed, a small fraction of the angular momentum would have been communicated to the condensing nucleus, and this may be the explanation of the rotation of the sun in a plane which does not differ very much from the invariable plane. It is suggested that after the main nebula collapsed into discrete condensations, stray wisps of the material composing the nebula, possessing random velocities, would hang about in conditions of unstable equilibrium between the stars in process of formation, and would finally be captured by one or the other of these. Here is a tentative explanation of the origin of the long-period comets. The theory is also able to explain the origin of the satellites and especially their direct motion, but it is admitted that retrograde satellites and also a system like that of Uranus present difficulties. Periodic comets may have been formed from matter left over from the planetary condensations.

No detailed explanation is attempted regarding the manner in which matter would be shed from the contracting mass, though certain possibilities are suggested. This particular part of the subject involves very abstruse mathematics dealing with gas-kinetics, and it is to be hoped that this branch will be developed further. It is conceivable that the theory may encounter some of its greatest difficulties here.

\section{HEALTH IN INDUSTRY}

$T$ HE importance of securing, in the crisis of manpower and woman-power through which Great Britain is now passing, that there is no waste of labour and that the full woman-power and manpower of the country is utilized to the maximum extent possible in furtherance of the nation's war effort-though it should be noted that the latter is not to be interpreted too narrowly-is now generally appreciated. As the last report of the Chief Inspector of Factories showed, the importance of eliminating the loss of man-hours and woman-hours due to accidents and to sickness is still far from being realized. Nor, as that report and successive reports of the Select Committee on National Expenditure have indicated, have managements either in industry or in Government departments and factories learnt the lessons and applied to good purpose the experience acquired during the War of 1914-18.

That truth is well emphasized in the third report of the 1942-43 session from the Select Committee on National Expenditure. Here the results of the investigations of the Committee on the health and welfare of women in war factories are detailed, and the observation recorded at the outset that little attention seems to have been paid to many of the constructive suggestions contained in earlier reports on health and welfare problems would justify this further inquiry. The relation of health to production is indeed one of the widest interest, as well as of growing importance as the demands of the War grow more imperative. It involves the co-operation of the worker as well as of factory management and of the Government.

Broadly speaking, an efficient general state of health in industry depends on the improvement and maintenance of what is commonly called physical fitness ; the prevention of diseases and of accidents ; the treatment of sick and injured persons and their rehabilitation to fit them for return to industry. The problem to-day is largely one of the most economical use of women, particularly in view of the breakdown of any system of medical examination as to the fitness of women for factory work before they are transferred to work away from home.

The results of this inquiry are set forth in three main divisions : health and welfare within the factory, and, secondly, outside the factory; and the State and industrial health. In regard to the first, matters relating to the maintenance of health can be grouped under three general headings : $(a)$ physical amenities, such as the provision of satisfactory canteens, cloakrooms, rest-rooms, etc. ; (b) medical provisions, such as first-aid, preventive measures for specific industrial diseases, and the general medical supervision of the 